\title{
REAL ALMOST ZEROS OF RANDOM POLYNOMIALS WITH COMPLEX COEFFICIENTS
}

\author{
K. FARAHMAND, A. GRIGORASH, AND P. FLOOD
}

Received 30 April 2004 and in revised form 14 July 2004

We present a simple formula for the expected number of times that a complex-valued Gaussian stochastic process has a zero imaginary part and the absolute value of its real part is bounded by a constant value $M$. We show that only some mild conditions on the stochastic process are needed for our formula to remain valid. We further apply this formula to a random algebraic polynomial with complex coefficients. We show how the above expected value in the case of random algebraic polynomials varies for different behaviour of $M$.

\section{Introduction}

There is a significant amount of work concerning the expected number of zeros of stationary normal processes. For the obvious difficulties that arise, less is known in the nonstationary case, see, for example, [2] and references therein. However, random polynomials are special cases of the latter processes and, therefore, their properties are of special interest. Also random polynomials with complex coefficients will introduce a new dimension to the study of nonstationary stochastic processes, caused by real and imaginary parts of polynomials.

In this work, we initially develop some properties of a complex-valued stochastic process in order to apply the result to random polynomials with complex coefficients. There are many mathematical interests in these types of polynomials, see [5,6]. Also the physical interest in general theory of random polynomials is described in the significant work of Edelman and Kostlan [4] which contains several new and interesting methods. The earlier results concerning various types of polynomials are reviewed in the comprehensive book of Bharucha-Reid and Sambandham [1]. Wilkins [8, 9] produced an analytical method which significantly improved the previous results.

As noted by Dunnage [3], for the case of random polynomials with complex coefficients, there can be no analogue of the asymptotic formula for the expected number of real zeros. Therefore, it is of special interest to study the events in which the imaginary part of the polynomial is zero and the real part is bounded by a constant $M$. To this end, a complex number $a(t)+\imath b(t)$ is called a real $M$-almost zero at $t=t_{0}$ if $b\left(t_{0}\right)=0$ and 
$\left|a\left(t_{0}\right)\right|<M$. On the other hand, it is called zero $M$-almost real if $a\left(t_{0}\right)=0$ and $\left|b\left(t_{0}\right)\right|<M$. In [5], the average number $E\{N(\alpha, \beta ; M)\}$ of real $M$-almost zeros of a complex-valued stochastic process $H(t)=\zeta(t)+\imath \xi(t)$ in the interval $t \in(\alpha, \beta)$ is given as

$$
E\{N(\alpha, \beta ; M)\}=\int_{\alpha}^{\beta} d t \int_{-M}^{M} d x \int_{-\infty}^{\infty}|z| p_{t}(x, 0, z) d z .
$$

In (1.1), $p_{t}(x, y, z)$ denotes the three-dimensional density function for the real part $\zeta(t)$ of the process, the imaginary part $\xi(t)$, and its quadratic mean derivative $\xi^{\prime}(t)$. The above formula holds for $\zeta(t)$ and $\xi(t)$ subject to conventional limitations:

(i) $\zeta(t)$ and $\xi(t)$ are real-valued separable processes;

(ii) they possess continuous sample paths with probability one; and

(iii) their joint distribution is nonsingular for each $t$;

(iv) $E\{\xi(t)\}$ has a continuous derivative for all $t$;

(v) $\operatorname{cov}(\xi(t), \xi(s))$ has a second mixed partial derivative which is continuous for all $t$ and $s$.

The above formula is then applied to the random algebraic polynomial

$$
\mathscr{P}(x)=\sum_{j=0}^{n-1}\left(a_{j} x^{j}+\imath b_{j} x^{j}\right)
$$

to obtain , with $n \rightarrow \infty$, the asymptotic approximation of the sought expected value. The polynomial coefficients $a_{j}+\imath b_{j}$ have real and imaginary parts $a_{j}$ and $b_{j}$ forming sequences of independent normal random variables. The work [5] dealt with the case when these random variables were all with mean 0 and variance 1 . In the present work, we will show that the case of arbitrary mean and variance is amenable to a similar, albeit more technically involved, asymptotic analysis.

\section{Gaussian complex-valued processes with independent real and imaginary parts}

In this section, we consider only Gaussian processes, and will show how formula (1.1) can be simplified in a particular case when $\zeta(t)$ and $\xi(t)$ are stochastically independent. We make no additional assumptions about the expected values or variances involved. The covariance matrix of the processes $\zeta(t), \xi(t)$, and $\xi^{\prime}(t)$ is

$$
\Sigma=\left(\begin{array}{ccc}
A^{2} & 0 & 0 \\
0 & B^{2} & F \\
0 & F & C^{2}
\end{array}\right)
$$

where

$$
A^{2}=\operatorname{var}\{\zeta(t)\}, \quad B^{2}=\operatorname{var}\{\xi(t)\}, \quad C^{2}=\operatorname{var}\left\{\xi^{\prime}(t)\right\}, \quad F=\operatorname{cov}\{\xi(t), \dot{\xi}(t)\} .
$$

By the assumption of nonsingularity,

$$
\Delta^{2}=B^{2} C^{2}-F^{2}
$$


is nonnegative. Denote also the expected values as follows:

$$
m_{1}=E\{\zeta(t)\}, \quad m_{2}=E\{\xi(t)\}, \quad m_{3}=E\left\{\xi^{\prime}(t)\right\} .
$$

Using our knowledge of the normal distribution, we expand the density $p_{t}(x, y, z)$, and a straightforward algebra transforms formula (1.1) to its "complicated" form

$$
\begin{aligned}
& E\{N(\alpha, \beta ; M)\} \\
& \quad=(2 \pi)^{-3 / 2} \int_{\alpha}^{\beta} \mathfrak{d}(t)\left(\int_{-M}^{M}\left\{\int_{-\infty}^{\infty}|z| \exp \left[-\frac{\mathfrak{A} x^{2}}{2}-\frac{\mathfrak{C} z^{2}}{2}+x \mathfrak{X}_{1}+z \mathfrak{X}_{2}-\frac{\mathfrak{X}_{0}}{2}\right] d z\right\} d x\right) d t,
\end{aligned}
$$

defining

$$
\begin{aligned}
\mathfrak{A}=\frac{1}{A^{2}}, \quad \mathfrak{B}=\frac{C^{2}}{\Delta^{2}}, & \mathfrak{C}=\frac{B^{2}}{\Delta^{2}}, \quad \mathfrak{F}=\frac{-F}{\Delta^{2}}, \\
\mathfrak{d}(t)=(\operatorname{det} \Sigma)^{-1 / 2}=(A \Delta)^{-1}, & \mathfrak{X}_{0}=m_{1}^{2} \mathfrak{A}+m_{2}^{2} \mathfrak{B}+m_{3}^{2} \mathfrak{C}+2 m_{1} m_{3} \mathfrak{F}, \\
\mathfrak{X}_{1}=m_{1} \mathfrak{A}, & \mathfrak{X}_{2}=m_{2} \mathfrak{F}+m_{3} \mathfrak{C} .
\end{aligned}
$$

We rewrite the expression inside the exponent of $z$ and $x$ in the form

$$
-a(x+f)^{2}-c(z+g)^{2}+h
$$

where $a, b, c, f, g$, and $h$ are expressions that depend neither on $z$ nor on $x$, but possibly on $t$. Equating the coefficients at the like terms gives

$$
\begin{aligned}
& a=\frac{\mathfrak{A}}{2}=\frac{1}{2 A^{2}}, \quad c=\frac{\mathfrak{C}}{2}=\frac{B^{2}}{2 \Delta^{2}}, \quad f=\frac{-\mathfrak{X}_{1}}{\mathfrak{A}}=-m_{1}, \\
& g=\frac{-\mathfrak{X}_{2}}{\mathfrak{C}}=\frac{m_{2} F}{B^{2}}-m_{3}, \quad h=\frac{m_{2}^{2}}{2}\left[\frac{\mathfrak{F}^{2}}{\mathfrak{C}}-\mathfrak{B}\right]=-\frac{m_{2}^{2}}{2 B^{2}} .
\end{aligned}
$$

With two further variable changes, $\zeta=z+g$ and $\tau=x+f$, formula (2.5) simplifies to

$$
E\{N(\alpha, \beta ; M)\}=(2 \pi)^{-3 / 2} \int_{\alpha}^{\beta} \mathfrak{d}(t) \exp (h) \int_{-\infty}^{\infty} \int_{f-M}^{f+M}|g-\zeta| \exp \left[-a \tau^{2}-c \zeta^{2}\right] d \tau d \zeta d t
$$

Now integration over $\tau$ and over $\zeta$ can be separated. Moreover, we remove the absolute value sign by partitioning the integration interval over $\zeta$ into two, about the point $\zeta=g$, such that (2.9) transforms to

$$
E\{N(\alpha, \beta ; M)\}=H_{1}+H_{2},
$$


where

$$
\begin{aligned}
& H_{1}=(2 \pi)^{-3 / 2} \int_{\alpha}^{\beta} \mathfrak{d}(t) \exp (h) g(t) \\
& \times\left(\int_{f-M}^{f+M} \exp \left[-a \tau^{2}\right] d \tau\right)\left(\int_{-g}^{g} \exp \left[-c \zeta^{2}\right] d \zeta\right) d t, \\
& H_{2}=(2 \pi)^{-3 / 2} \int_{\alpha}^{\beta} \mathfrak{d}(t) \exp (h)\left(\int_{f-M}^{f+M} \exp \left[-a \tau^{2}\right] d \tau\right) \\
& \quad \times\left(\int_{g}^{\infty} \zeta \exp \left[-c \zeta^{2}\right] d \zeta-\int_{-\infty}^{g} \zeta \exp \left[-c \zeta^{2}\right] d \zeta\right) d t .
\end{aligned}
$$

It turns out that $H_{1}$ defined in (2.11) is a bounded quantity that does not contribute anything significant to the asymptotic estimate of the expected number of real $M$-almost zeros in Theorem 3.1 in the next section. This effect is similar to that in [7] and is achieved through a similar statement that follows.

Lemma 2.1. If $\lim _{t \rightarrow \alpha}\left(m_{2} / B\right)$ and $\lim _{t \rightarrow \beta}\left(m_{2} / B\right)$ exist as finite or infinite, and if $\xi$ and $\zeta$ satisfy the above conditions for formula (2.10) to hold, then the component $H_{1}$ in this formula is bounded by a constant

$$
H_{1}=O(1)
$$

Proof. From (2.11), after some algebra,

$$
\begin{aligned}
H_{1}=(2 \pi)^{-3 / 2} \int_{\alpha}^{\beta} A^{-1} \Delta^{-1} \exp \left(-\frac{m_{2}^{2}}{2 B^{2}}\right)\left(m_{2} \frac{F}{B^{2}}-m_{3}\right) \\
\quad \times\left(\int_{-m_{1}-M}^{-m_{1}+M} \exp \left[-\frac{\tau^{2}}{2 A^{2}}\right] d \tau\right)\left(\int_{-g}^{g} \exp \left[-\frac{B^{2}}{2 \Delta^{2}} \zeta^{2}\right] d \zeta\right) d t .
\end{aligned}
$$

Notice that

$$
\left|\int_{-g}^{g} \exp \left[-\frac{B^{2}}{2 \Delta^{2}} \zeta^{2}\right] d \zeta\right| \leq \frac{\Delta \sqrt{2 \pi}}{B}, \quad\left|\int_{-m_{1}-M}^{-m_{1}+M} \exp \left[-\frac{\tau^{2}}{2 A^{2}}\right] d \tau\right| \leq A \sqrt{2 \pi} .
$$

Thus, from (2.14),

$$
\left|H_{1}\right| \leq(2 \pi)^{-1 / 2}\left|\int_{\alpha}^{\beta} B^{-1}\left(\frac{m_{2} F}{B^{2}}-m_{3}\right) \exp \left(-\frac{m_{2}^{2}}{2 B^{2}}\right) d t\right| .
$$

By assumption (iv), $m_{2}$ is differentiable, and differentiation over $t$ can be exchanged with taking the expected value (essentially integration over $\xi$ )

$$
\frac{d\left(m_{2}\right)}{d t}=E\left(\frac{d \xi}{d t}\right)=m_{3}
$$


By assumption $(\mathrm{v}), d(\operatorname{var}(\xi)) / d t$ exists, and again differentiation can be interchanged with taking the expected value, such that

$$
\frac{d(\operatorname{var}(\xi))}{d t}=2\left(E\left(\xi \xi^{\prime}\right)-m_{2} m_{3}\right)=2 F
$$

Also by definition of $B^{2}$, it is easy to show that

$$
\frac{d B}{d t}=\frac{F}{B}
$$

We change the integration variable in (2.16) from $t$ to $u=m_{2} / B$. Then from (2.17) and (2.19), $d u=B^{-1}\left(m_{3}-m_{2} F B^{-2}\right) d t$. With the new variable, inequality (2.16) becomes

$$
\left|H_{1}\right| \leq \frac{1}{\sqrt{2 \pi}}\left|-\int_{u_{\alpha}}^{u_{\beta}} \exp \left(-\frac{u^{2}}{2}\right) d u\right|=\left|\Phi\left(u_{\alpha}\right)-\Phi\left(u_{\beta}\right)\right| \leq 1=O(1)
$$

where $\Phi(x)=(2 \pi)^{-1 / 2} \int_{-\infty}^{x} \exp \left(-s^{2} / 2\right) d s$ is the standard normal distribution function, $u_{\alpha}=\lim _{t \rightarrow \alpha}\left(m_{2} / B\right)$, and $u_{\beta}=\lim _{t \rightarrow \beta}\left(m_{2} / B\right)$. The limits exist by assumption, the lemma is proved.

\section{Random algebraic polynomials}

In this section, we apply formula (2.10) and Lemma 2.1 to estimate the expected number of real $M$-almost zeros of polynomial

$$
P(x)=\sum_{j=0}^{n}\left(a_{j} x^{j}+\imath b_{j} x^{j}\right)=F(x)+\imath G(x)
$$

in real variable $x$ with complex-valued coefficients $a_{j}+\imath b_{j}$, where $a_{j}$ and $b_{j}$ are all independent real Gaussian random variables. Denote their expected values

$$
E\left(a_{j}\right)=\mu, \quad E\left(b_{j}\right)=\nu,
$$

where $\mu$ and $\nu$ are, generally speaking, different values and both are nonzero. Denote variances

$$
\operatorname{var}\left(a_{j}\right)=\sigma^{2}, \quad \operatorname{var}\left(b_{j}\right)=v^{2},
$$

and assume that they are, in general, nonunit and not equal to each other.

THeOREм 3.1. If the coefficients in polynomial (3.1) are defined as above, and if there exists a constant $k>1$ such that $M / \sigma=o\left(n^{k}\right)$, then

(A) for $M / \sigma=o(\ln n)$,

$$
E\{N(-\infty, \infty ; M)\}=o(\ln n)
$$


(B) for $(n / \ln n)=o\left(v^{2} / v^{2}\right)$,

$$
E\{N(-\infty, \infty ; M)\}=o(\ln n)
$$

(C) and for $v^{2} / v^{2}=o\left(n^{-1} \ln n\right), \sqrt{n / \ln n}=o(M / \sigma)$, and $\mu / \sigma=O\left(\sqrt{n^{-1} \ln n}\right)$,

$$
E\{N(-\infty, \infty ; M)\} \sim \pi^{-1} \ln n .
$$

3.1. Proof of Theorem 3.1. First of all, we rewrite $\mathrm{H}_{2}$ in terms of the random polynomial (3.1). From definition (2.12), after some algebra,

$$
\begin{aligned}
H_{2}=(2 \pi)^{-3 / 2} \int_{\alpha}^{\beta} & (A \Delta)^{-1} \exp \left(-\frac{m_{2}^{2}}{2 B^{2}}\right)\left(\int_{-m_{1}-M}^{-m_{1}+M} \exp \left[-\frac{\tau^{2}}{2 A^{2}}\right] d \tau\right) \\
& \times\left(\int_{g}^{\infty} \zeta \exp \left[-\frac{B^{2}}{2 \Delta^{2}} \zeta^{2}\right] d \zeta-\int_{-\infty}^{g} \zeta \exp \left[-\frac{B^{2}}{2 \Delta^{2}} \zeta^{2}\right] d \zeta\right) d x
\end{aligned}
$$

The above integrals over $\zeta$ can be taken, and the integral over $\tau$ can be expressed in terms of the standard normal distribution function.

$$
H_{2}=\pi^{-1} \int_{\alpha}^{\beta} \frac{\Delta}{B^{2}} \exp (-\mathfrak{T}(x))\left[\Phi\left(\mathfrak{S}^{+}(x)\right)-\Phi\left(\mathfrak{S}^{-}(x)\right)\right] d x,
$$

where

$$
\begin{aligned}
\mathfrak{T}(x) & =\frac{m_{2}^{2}}{2 B^{2}}+\frac{B^{2}}{2 \Delta^{2}}\left(\frac{m_{2} F}{B^{2}}-m_{3}\right)^{2}, \\
\mathfrak{S}^{+}(x) & =-\frac{m_{1}+M}{A}, \quad \mathfrak{S}^{-}(x)=-\frac{m_{1}-M}{A} .
\end{aligned}
$$

By Lemma 2.1 and formula (2.10), we have

$$
E\{N(-\infty, \infty ; M)\}=H_{2}+O(1)
$$

hence the evaluation of the sought expected value is reduced to the evaluation of $H_{2}$, in (3.8). For clarity, we list the expected values and covariances involved in the following:

$$
\begin{gathered}
m_{1}=\mu \frac{x^{n+1}-1}{x-1}, \quad m_{2}=v \frac{x^{n+1}-1}{x-1}, \quad m_{3}=v \frac{n x^{n+1}-n x^{n}-x^{n}+1}{(x-1)^{2}}, \\
A^{2}=\frac{\sigma^{2}\left(x^{2 n+2}-1\right)}{x^{2}-1}, \quad B^{2}=\frac{v^{2}\left(x^{2 n+2}-1\right)}{x^{2}-1}, \\
C^{2}=v^{2}\left\{\frac{n^{2} x^{2 n}}{x^{2}-1}-\frac{2 n x^{2 n}}{\left(x^{2}-1\right)^{2}}+\frac{\left(1+x^{2}\right)\left(x^{2 n}-1\right)}{(x 2-1)^{3}}\right\}, \\
F=v^{2}\left\{\frac{n x^{2 n+1}}{x^{2}-1}+\frac{x\left(1-x^{2 n}\right)}{\left(x^{2}-1\right)^{2}}\right\} .
\end{gathered}
$$


These values form the components inside formula (3.8), as follows. From (2.3),

$$
\Delta^{2}=\frac{v^{4}}{\left(x^{2}-1\right)^{4}}\left\{\left(1-x^{2 n+2}\right)^{2}-(n+1)^{2} x^{2 n}\left(x^{2}-1\right)^{2}\right\} .
$$

From (3.10),

$$
\mathfrak{S}^{+}(x)=\left(\frac{M}{\sigma}-\frac{\mu}{\sigma} V(x)\right) U(x), \quad \mathfrak{S}^{-}(x)=\left(-\frac{M}{\sigma}-\frac{\mu}{\sigma} V(x)\right) U(x),
$$

where

$$
U(x)=\sqrt{\frac{x^{2}-1}{x^{2 n+2}-1}}, \quad V(x)=\frac{x^{n+1}-1}{x-1} .
$$

Also

$$
\frac{\Delta}{B^{2}}=\frac{\sqrt{\left(1-x^{2 n+2}\right)^{2}-(n+1)^{2} x^{2 n}\left(x^{2}-1\right)^{2}}}{\left(x^{2}-1\right)\left(x^{2 n+2}-1\right)} .
$$

Therefore with the above formulae, formula (3.9) results in

$$
\mathfrak{T}(x)=\frac{v^{2}(x+1)\left(x^{n+1}-1\right)^{2}}{v^{2}(x-1)\left[x^{2 n+2}-1+(n+1) x^{n}\left(x^{2}-1\right)\right]} .
$$

We proceed by splitting the $x$ axis into several intervals, and attaining estimates on each of them in turn. Let $\varepsilon \equiv \varepsilon(n)=n^{\gamma-1}$, where $\gamma=\ln (k \ln n)(\ln n)^{-1}$, and $k$ is the constant from the theorem formulation. In other words, $\varepsilon=k \ln n / n$. It is easy to show that $\varepsilon$ has the following properties:

$$
\begin{gathered}
\lim _{n \rightarrow \infty} \varepsilon(n)=0, \\
\sqrt{n \varepsilon(n)}=n^{\gamma / 2}=\sqrt{k \ln n}=o(\ln n), \\
n(1-\varepsilon)^{n} \longrightarrow n \exp (-n \varepsilon)=n \exp (-k \ln n)=n^{1-k} \longrightarrow 0, \\
(1+\varepsilon)^{-n} \longrightarrow \exp (-n \varepsilon) \longrightarrow 0 \quad \text { as } n \longrightarrow \infty .
\end{gathered}
$$

3.1.1. Negative tail: interval $-\infty<x<-1-\varepsilon$. In this interval, it is clear that $\mathfrak{T}(x)>0$. Then $\exp (-\mathfrak{T}(x))<1$, and

$$
\left|H_{2}\right|<\pi^{-1}\left|\int_{-\infty}^{-1-\varepsilon} \frac{\Delta}{B^{2}}\left[\Phi\left(\mathfrak{S}^{+}(x)\right)-\Phi\left(\mathfrak{S}^{-}(x)\right)\right] d x\right| .
$$

Changing the integration variable to $y=-x^{-1}$ transforms this inequality to

$$
\left|H_{2}\right|<\pi^{-1}\left|\int_{0}^{(1+\varepsilon)^{-1}} \frac{\Delta(1 / y)}{y^{2} B^{2}(1 / y)}\left[\Phi\left(\mathfrak{S}^{+}\left(\frac{1}{y}\right)\right)-\Phi\left(\mathfrak{S}^{-}\left(\frac{1}{y}\right)\right)\right] d y\right|,
$$


where

$$
\begin{gathered}
\frac{\Delta}{y^{2} B^{2}}=\frac{1}{1-y^{2}} \sqrt{1-\frac{(n+1)^{2} y^{2 n}\left(1-y^{2}\right)^{2}}{\left(1-y^{2 n+2}\right)^{2}}}, \\
\Phi\left(\mathfrak{S}^{+}\right)-\Phi\left(\mathfrak{S}^{-}\right)=\frac{1}{\sqrt{2 \pi}} \int_{(-M U-\mu V U) / \sigma}^{(M U-\mu V U) / \sigma} \exp \left(-\frac{\zeta^{2}}{2}\right) d \zeta .
\end{gathered}
$$

Changing variable $\zeta=s-\mu V U / \sigma$, where $U$ and $V$ are given in (3.15), in the last expression produces

$$
\begin{aligned}
\Phi\left(\mathfrak{S}^{+}\right)-\Phi\left(\mathfrak{S}^{-}\right) & =\frac{2}{\sqrt{2 \pi}} \int_{0}^{M U / \sigma} \exp \left(-\frac{1}{2}\left\{s-\frac{\mu}{\sigma} V U\right\}^{2}\right) d s \\
& \leq \sqrt{\frac{2}{\pi}} \int_{0}^{M U / \sigma} d s=\frac{M}{\sigma} \sqrt{\frac{2\left(1-y^{2}\right) y^{2 n}}{\pi\left(1-y^{2 n+2}\right)}} .
\end{aligned}
$$

Notice that in this interval $y<1$, and since

$$
\frac{1-y^{2 n+2}}{1-y^{2}}=\sum_{j=1}^{n+1} y^{2(j-1)}<n+1
$$

shows that (3.24) is bounded,

$$
\left|\frac{\Delta(y)}{y^{2} B^{2}(y)}\right|<\frac{\sqrt{1-y^{2 n}}}{1-y^{2}} .
$$

With (3.26) and (3.28), inequality (3.23) can be strengthened and estimated as follows:

$$
\begin{aligned}
\left|H_{2}\right| & <\frac{2}{\pi \sqrt{2 \pi}} \frac{M}{\sigma}\left|\int_{0}^{(1+\varepsilon)^{-1}} \frac{\Delta U}{y^{2} B^{2}} d y\right| \\
& <\frac{2}{\pi \sqrt{2 \pi}} \frac{M}{\sigma}\left|\int_{0}^{(1+\varepsilon)^{-1}} \sqrt{\frac{y^{2 n}}{1-y^{2}}} d y\right| \\
& <\frac{2}{\pi \sqrt{2 \pi}} \frac{M}{\sigma}(1+\varepsilon)^{-n}\left|\int_{0}^{(1+\varepsilon)^{-1}} \frac{1}{\sqrt{1-y^{2}}} d y\right| \\
& <\frac{1}{\sqrt{2 \pi}} \frac{M}{\sigma}(1+\varepsilon)^{-n} \longrightarrow \frac{1}{\sqrt{2 \pi}} \frac{M}{\sigma} n^{-k} \longrightarrow 0,
\end{aligned}
$$

as $n \rightarrow \infty$. Therefore

$$
E\{N(-\infty,-1-\varepsilon ; M)\}=O(1) .
$$

3.1.2. Neighbourhood of -1 : interval $-1-\varepsilon \leq x \leq-1+\varepsilon$. As in the previous interval, $\exp (-\mathfrak{T}(x))<1$ and $\Phi\left(\mathfrak{S}^{+}\right)-\Phi\left(\mathfrak{S}^{-}\right)<1$, both expressions approaching 1 in the limit 
with $x \rightarrow-1$. Hence from (3.8),

$$
\begin{aligned}
\left|H_{2}\right|< & \pi^{-1}\left|\int_{-1-\varepsilon}^{-1} \frac{\Delta}{B^{2}} d x\right|+\pi^{-1}\left|\int_{-1}^{-1+\varepsilon} \frac{\Delta}{B^{2}} d x\right| \\
= & \pi^{-1}\left|\int_{(1+\varepsilon)^{-1}}^{1} \frac{1}{1-y^{2}} \sqrt{1-\frac{(n+1)^{2} y^{2 n}\left(1-y^{2}\right)^{2}}{\left(1-y^{2 n+2}\right)^{2}}} d y\right| \\
& +\pi^{-1}\left|\int_{1-\varepsilon}^{1} \frac{1}{1-x^{2}} \sqrt{1-\frac{(n+1)^{2} x^{2 n}\left(1-x^{2}\right)^{2}}{\left(1-x^{2 n+2}\right)^{2}}} d x\right| .
\end{aligned}
$$

Here, the variable change $y=-1 / x$ and (3.24) were used in the interval $x \in[-1-\varepsilon,-1]$, and the fact that $\Delta / B^{2}$ given in (3.16) is an even function was used in the interval $x \in$ $[-1,-1+\varepsilon]$.

From the geometric progression sum, for all $x \leq 1$, we have $1-x^{2 n+2} \leq(2 n+2)(1-x)$. Therefore,

$$
\frac{(n+1)^{2} x^{2 n}\left(1-x^{2}\right)^{2}}{\left(1-x^{2 n+2}\right)^{2}} \geq \frac{x^{2 n}(1+x)^{2}}{4}
$$

This immediately yields

$$
\begin{aligned}
1-\frac{(n+1)^{2} x^{2 n}\left(1-x^{2}\right)^{2}}{\left(1-x^{2 n+2}\right)^{2}} & \\
\leq & \left(1-\frac{x^{n}(1+x)}{2}\right)\left(1+\frac{x^{n}(1+x)}{2}\right) \leq 2-x^{n}(1+x) \\
& =\left(1-x^{n}\right)+\left(1-x^{n+1}\right) \leq(n+n+1)(1-x) .
\end{aligned}
$$

Also from (3.31) and (3.33),

$$
\begin{aligned}
\left|H_{2}\right| & <\frac{\sqrt{2 n+1}}{\pi}\left\{\left|\int_{(1+\varepsilon)^{-1}}^{1} \frac{d y}{\sqrt{1-y}}\right|+\left|\int_{1-\varepsilon}^{1} \frac{d x}{\sqrt{1-x}}\right|\right\} \\
& =\frac{2}{\pi} \sqrt{2+\frac{1}{n}}\left\{\frac{1}{\sqrt{1+\varepsilon}}+1\right\} \sqrt{k \ln n}=O(\sqrt{\ln n})=o(\ln n) .
\end{aligned}
$$

Thereby, we have proved that

$$
E\{N(-1-\varepsilon,-1+\varepsilon ; M)\}=o(\ln n)
$$

3.1.3. Neighbourhood of +1 and the positive tail: intervals $1-\varepsilon \leq x \leq 1+\varepsilon$ and $1+\varepsilon<$ $x<+\infty$. Since $\Delta / B^{2}$ given in (3.16) is an even function, and the upper boundaries in the inequalities (3.26) and (3.28) are even functions too, the reasoning on these intervals repeats entirely that on the previous two intervals, with $y=1 / x$ variable change for $x \geq 1$. Therefore,

$$
E\{N(1-\varepsilon, 1+\varepsilon ; M)\}=o(\ln n), \quad E\{N(1+\varepsilon, \infty ; M)\}=O(1) .
$$


3.1.4. Main negative interval $-1+\varepsilon<x \leq 0$. This interval turns out to contain, on average, most of the real $M$-almost zeros. The estimates here depend on the behaviour of ratios $M / \sigma, \mu / \sigma$, and $\nu / v$ with respect to the growth of $n$. We therefore break our analysis into several distinct cases that produce different results.

Case $A(M / \sigma=o(\ln n))$. That is, either $M / \sigma$ is a constant or this ratio grows "much slower" than $n$ if it at all depends on $n$. Similarly to Section 3.1.1,

$$
\left|H_{2}\right|<\pi^{-1}\left|\int_{-1+\varepsilon}^{0} \frac{\Delta}{B^{2}}\left[\Phi\left(\mathfrak{S}^{+}(x)\right)-\Phi\left(\mathfrak{S}^{-}(x)\right)\right] d x\right| .
$$

We also observe that, like (3.26) and (3.28),

$$
\Phi\left(\mathfrak{S}^{+}\right)-\Phi\left(\mathfrak{S}^{-}\right) \leq \frac{M}{\sigma} \sqrt{\frac{2\left(1-x^{2}\right)}{\pi\left(1-x^{2 n+2}\right)}}, \quad\left|\frac{\Delta}{B^{2}}\right|<\frac{\sqrt{1-x^{2 n}}}{1-x^{2}},
$$

respectively. Hence,

$$
\begin{aligned}
\left|H_{2}\right| & <\frac{2}{\pi \sqrt{2 \pi}} \frac{M}{\sigma}\left|\int_{-1+\varepsilon}^{0} \frac{\Delta U}{B^{2}} d x\right| \\
& <\frac{2}{\pi \sqrt{2 \pi}} \frac{M}{\sigma}\left|\int_{-1+\varepsilon}^{0} \sqrt{\frac{\left(1-x^{2 n}\right)}{\left(1-x^{2}\right)\left(1-x^{2 n+2}\right)}} d x\right| \\
& <\frac{2}{\pi \sqrt{2 \pi}} \frac{M}{\sigma}\left|\int_{-1+\varepsilon}^{0} \frac{d x}{\sqrt{1-x^{2}}}\right|<\frac{M}{\sigma \sqrt{2 \pi}} .
\end{aligned}
$$

Therefore, for the above assumptions,

$$
E\{N(-1+\varepsilon, 0 ; M)\}=o(\ln n) .
$$

This concludes Case A on this interval.

To proceed with other cases, we first notice that in the present interval, the inequality $\left|(n+1) x^{n}\right|<(n+1)(1-\varepsilon)^{n}$ holds, and by (3.20) the quantity $\left|(n+1) x^{n}\right|$ tends to 0 with $n \rightarrow \infty$. Hence by (3.16), (3.17) and the principle of compressed variable,

$$
\mathfrak{T}(x) \longrightarrow \frac{v^{2}(x+1)}{v^{2}(1-x)}, \quad \frac{\Delta}{B^{2}} \longrightarrow \frac{1}{\left(1-x^{2}\right)} .
$$

By the same principle, (3.14) and (3.15) give

$$
\begin{aligned}
& \mathfrak{S}^{+}(x) \longrightarrow\left(\frac{M}{\sigma}-\frac{\mu}{\sigma(1-x)}\right) \sqrt{1-x^{2}} \\
& \mathfrak{S}^{-}(x) \longrightarrow\left(-\frac{M}{\sigma}-\frac{\mu}{\sigma(1-x)}\right) \sqrt{1-x^{2}} .
\end{aligned}
$$

Changing the integration variable to $y=-x$ in (3.8) and using the above expressions gives

$$
H_{2} \longrightarrow \int_{0}^{1-\varepsilon} O_{1}(y) d y-\int_{0}^{1-\varepsilon} O_{2}(y) d y
$$


where

$$
\begin{aligned}
& \int_{0}^{1-\varepsilon} O_{1}(y) d y=\frac{1}{\pi} \int_{0}^{1-\varepsilon} \frac{1}{1-y^{2}} \exp \left(-\frac{v^{2}(1-y)}{v^{2}(1+y)}\right) \Phi\left[\left(\frac{M}{\sigma}-\frac{\mu}{\sigma(1+y)}\right) \sqrt{1-y^{2}}\right] d y, \\
& \int_{0}^{1-\varepsilon} O_{2}(y) d y=\frac{1}{\pi} \int_{0}^{1-\varepsilon} \frac{1}{1-y^{2}} \exp \left(-\frac{v^{2}(1-y)}{v^{2}(1+y)}\right) \Phi\left[\left(-\frac{M}{\sigma}-\frac{\mu}{\sigma(1+y)}\right) \sqrt{1-y^{2}}\right] d y .
\end{aligned}
$$

Now we need a quantity $\delta(n)$ such that $\varepsilon(n)<\delta(n)<1, \delta(n) \rightarrow 0$ and $\ln \left(\delta^{-1}(n)\right)=$ $o\left(\ln \left(\varepsilon^{-1}(n)\right)\right)$ as $n \rightarrow \infty$. That is, $\delta$ can be any infinitesimal that bounds $\varepsilon$ from above, and such that $\ln \delta$ is of order less than $\ln \varepsilon$ with $n \rightarrow \infty$. We choose $\delta=(\ln n)^{-1}$, which obviously satisfies these conditions. Then,

$$
\begin{gathered}
\int_{1-\delta}^{1-\varepsilon} O_{1}(y) d y \leq \int_{0}^{1-\varepsilon} O_{1}(y) d y \leq \int_{1-\delta}^{1-\varepsilon} O_{1}(y) d y+\pi^{-1} \int_{0}^{1-\delta} \frac{d y}{1-y^{2}}, \\
-\int_{1-\delta}^{1-\varepsilon} O_{2}(y) d y-\pi^{-1} \int_{0}^{1-\delta} \frac{d y}{1-y^{2}} \leq-\int_{0}^{1-\varepsilon} O_{2}(y) d y \leq-\int_{1-\delta}^{1-\varepsilon} O_{2}(y) d y .
\end{gathered}
$$

Also notice that

$$
\pi^{-1} \int_{0}^{1-\delta} \frac{d y}{1-y^{2}}=(2 \pi)^{-1} \ln \left(\frac{2-\delta}{\delta}\right)=O\left(\ln \left(\delta^{-1}\right)\right)=o(\ln n) .
$$

Hence by adding the two above inequalities, we have

$$
\begin{aligned}
& \int_{1-\delta}^{1-\varepsilon}\left(O_{1}(y)-O_{2}(y)\right) d y-O(\ln (\ln n)) \\
& \quad \leq \int_{0}^{1-\varepsilon}\left(O_{1}(y)-O_{2}(y)\right) d y \leq \int_{1-\delta}^{1-\varepsilon}\left(O_{1}(y)-O_{2}(y)\right) d y+O(\ln (\ln n)) .
\end{aligned}
$$

In other words, using (3.44), we have

$$
H_{2} \longrightarrow \int_{1-\delta}^{1-\varepsilon}\left(O_{1}(y)-O_{2}(y)\right) d y+O(\ln (\ln n))
$$

with $n \rightarrow \infty$. Therefore noticing that

$$
\begin{aligned}
\pi^{-1} \int_{1-\delta}^{1-\varepsilon} \frac{d y}{1-y^{2}} & =(2 \pi)^{-1} \ln \left|\frac{y+1}{y-1}\right|_{1-\delta}^{1-\varepsilon} \longrightarrow(2 \pi)^{-1}\left[\ln \left(2 \varepsilon^{-1}\right)-\ln \left(2 \delta^{-1}\right)\right] \\
& =(2 \pi)^{-1}[\ln (n)-\ln (k)]=(2 \pi)^{-1} \ln n+O(1)
\end{aligned}
$$

and using the mean value theorem in (3.49) leads to

$$
\begin{aligned}
H_{2} \longrightarrow & {\left[\Phi\left\{\left(\frac{M}{\sigma}-\frac{\mu}{\sigma\left(1+y_{*}\right)}\right) \sqrt{1-y_{*}^{2}}\right\}-\Phi\left\{\left(-\frac{M}{\sigma}-\frac{\mu}{\sigma\left(1+y_{*}\right)}\right) \sqrt{1-y_{*}^{2}}\right\}\right] } \\
& \times \exp \left(-\frac{v^{2}}{v^{2}} \frac{1-y_{*}}{1+y_{*}}\right) \frac{\ln n}{2 \pi}+O(\ln (\ln n)),
\end{aligned}
$$


where $y_{*}$ is the mean value theorem constant in the range $1-\delta<y_{*}<1-\varepsilon$. Since both $\delta$ and $\varepsilon$ tend to 0 as $n$ increases, (3.51) can be strengthened as

$$
\begin{aligned}
H_{2} \longrightarrow & {\left[\Phi\left\{\left(\frac{M}{\sigma}-\frac{\mu}{2 \sigma}\right) \sqrt{2 s_{*}}\right\}-\Phi\left\{\left(-\frac{M}{\sigma}-\frac{\mu}{2 \sigma}\right) \sqrt{2 s_{*}}\right\}\right] } \\
& \times \exp \left(-\frac{v^{2}}{v^{2}} \frac{s_{*}}{2}\right) \frac{\ln n}{2 \pi}+O(\ln (\ln n)),
\end{aligned}
$$

where $\varepsilon<s_{*}<\delta$ and $s_{*}=1-y_{*}$.

One can see that depending on the character of the asymptotic behaviour of the parameters involved, equation (3.52) can produce various values ranging from $\mathrm{H}_{2} \rightarrow$ $O(\ln (\ln n))$ to $H_{2} \simeq(2 \pi)^{-1} \ln n$. The rest of our analysis in the present interval is to consider several particular cases of the parameter combinations that yield various special cases of (3.52).

Case $B\left((n / \ln n)=o\left(v^{2} / v^{2}\right)\right)$. That is, $v^{2} / v^{2}$ is assumed to be growing faster than $n / \ln n$. Then $\left(v^{2} / v^{2}\right) s_{*}>\left(v^{2} / v^{2}\right) \varepsilon=\left(v^{2} / v^{2}\right)(k \ln n) / n \rightarrow+\infty$, and so

$$
\exp \left(-\frac{s_{*} v^{2}}{2 v^{2}}\right) \longrightarrow 0
$$

as $n \rightarrow \infty$. Since the quantity in square brackets in (3.52) is bounded, we thereby arrive to $H_{2} \rightarrow o(\ln n)+O(\ln (\ln n))$, and

$$
E\{N(-1+\varepsilon, 0 ; M)\}=o(\ln n),
$$

and this concludes Case B on this interval.

Case $C\left(v^{2} / v^{2}=o\left(n^{-1} \ln n\right), \sqrt{n / \ln n}=o(M / \sigma), \mu / \sigma=O\left(\sqrt{n^{-1} \ln n}\right)\right)$. Here,

$$
\frac{v^{2}}{v^{2}} s_{*}<\frac{v^{2}}{v^{2}} \delta=\frac{v^{2}}{v^{2} \ln n}=o\left(n^{-1}\right) \longrightarrow 0,
$$

and thus

$$
\exp \left(-\frac{v^{2}}{v^{2}} \frac{s_{*}}{2}\right) \longrightarrow 1
$$

as $n \rightarrow \infty$. Then instead of (3.52), we have

$$
H_{2} \longrightarrow\left[\Phi\left\{\left(\frac{M \sqrt{2 s_{*}}}{\sigma}-\frac{\mu \sqrt{2 s_{*}}}{2 \sigma}\right)\right\}-\Phi\left\{\left(-\frac{M \sqrt{2 s_{*}}}{\sigma}-\frac{\mu \sqrt{2 s_{*}}}{2 \sigma}\right)\right\}\right] \frac{\ln n}{2 \pi}+O(\ln (\ln n)) .
$$

From the condition on $M / \sigma$, we have

$$
\frac{M \sqrt{2 s_{*}}}{\sigma}>\frac{M \sqrt{2 \varepsilon}}{\sigma}=\frac{M \sqrt{2 k \ln n}}{\sigma \sqrt{n}} \longrightarrow+\infty .
$$

In turn, from the condition on $\mu / \sigma$, we have

$$
\frac{|\mu| \sqrt{2 s_{*}}}{\sigma}<\frac{|\mu| \sqrt{2 \delta}}{\sigma}=\frac{|\mu| \sqrt{2}}{\sigma \sqrt{\ln n}}=O\left(n^{-1 / 2}\right) \longrightarrow 0 .
$$


With (3.58) and (3.59), it is easy to see that the expression in square brackets in (3.57) tends to $\Phi(+\infty)-\Phi(-\infty)=1$, and therefore

$$
E\{N(-1+\varepsilon, 0 ; M)\} \sim \frac{\ln n}{2 \pi}
$$

Case $\mathrm{C}$ is concluded on this interval, and hence we have no more cases to consider.

3.1.5. Main positive interval $0<x<1-\varepsilon$. On this interval, the analysis borrows a lot from the analysis on its negative counterpart above. In order to avoid repetition, we only highlight the generations necessary.

Case $A(M / \sigma=o(\ln n))$. Since all the estimates in Case A above are even, we arrive at the symmetric conclusion

$$
E\{N(0,1-\varepsilon ; M)\}=o(\ln n)
$$

and Case A is concluded.

Also formulae (3.41), (3.42), and (3.43) hold exactly as above. The quantity $\delta$ remains the same. By the same reasoning in terms of $x$ as we did in terms of $y$, a sequence of expressions similar to those obtained in (3.44), (3.49), and (3.51) is produced. These easily yield

$$
\begin{aligned}
H_{2} \longrightarrow & {\left[\Phi\left\{\left(\frac{M}{\sigma}-\frac{\mu}{\sigma\left(1-x_{*}\right)}\right) \sqrt{1-x_{*}^{2}}\right\}-\Phi\left\{\left(-\frac{M}{\sigma}-\frac{\mu}{\sigma\left(1-x_{*}\right)}\right) \sqrt{1-x_{*}^{2}}\right\}\right] } \\
& \times \exp \left(-\frac{v^{2}}{v^{2}} \frac{1+x_{*}}{1-x_{*}}\right) \frac{\ln n}{2 \pi}+O(\ln (\ln n)),
\end{aligned}
$$

where $x_{*}$ is the mean value theorem constant from the range $1-\delta<x_{*}<1-\varepsilon$. The subtle difference between formulae (3.51) and (3.62) is the location of singularities. With $s_{*}=1-x_{*},(3.62)$ becomes

$$
\begin{aligned}
H_{2} \longrightarrow & {\left[\Phi\left\{\left(\frac{M}{\sigma}-\frac{\mu}{\sigma s_{*}}\right) \sqrt{2 s_{*}}\right\}-\Phi\left\{\left(-\frac{M}{\sigma}-\frac{\mu}{\sigma s_{*}}\right) \sqrt{2 s_{*}}\right\}\right] } \\
& \times \exp \left(-\frac{v^{2}}{v^{2}} \frac{2}{s_{*}}\right) \frac{\ln n}{2 \pi}+O(\ln (\ln n)) .
\end{aligned}
$$

In contrast to its counterpart (3.52), formula (3.63) contains infinitesimals in denominators. Nevertheless, as we show below, this does not hamper analysis of the two remaining cases.

Case $B\left((n / \ln n)=o\left(v^{2} / v^{2}\right)\right)$. Clearly, $\left(v^{2} / v^{2}\right) n^{-1} \ln n \rightarrow \infty$ and furthermore $\left(v^{2} / v^{2}\right)$ $\ln n \rightarrow \infty$. Then

$$
\frac{v^{2}}{v^{2}} s_{*}^{-1}>\frac{v^{2}}{v^{2}} \delta^{-1}=\frac{v^{2}}{v^{2}} \ln n \longrightarrow \infty, \quad \text { and so } \quad \exp \left(-\frac{v^{2}}{v^{2}} \frac{2}{s_{*}}\right) \longrightarrow 0,
$$


leading to

$$
E\{N(0,1-\varepsilon ; M)\}=o(\ln n) .
$$

This concludes Case B on this interval.

Case $C\left(v^{2} / v^{2}=o\left(n^{-1} \ln n\right), \sqrt{n / \ln n}=o(M / \sigma), \mu / \sigma=O\left(\sqrt{n^{-1} \ln n}\right)\right)$. Here,

$$
\frac{v^{2}}{v^{2} s_{*}}<\frac{v^{2}}{v^{2} \varepsilon}=\frac{v^{2} / v^{2}}{k n^{-1} \ln n} \longrightarrow 0, \text { and thus } \quad \exp \left(-\frac{v^{2}}{v^{2}} \frac{2}{s_{*}}\right) \longrightarrow 1
$$

as $n \rightarrow \infty$. Then instead of (3.57), we have

$$
\begin{aligned}
H_{2} \longrightarrow & {\left[\Phi\left\{\left(\frac{M \sqrt{2 s_{*}}}{\sigma}-\frac{\mu}{\sigma} \sqrt{\frac{2}{s_{*}}}\right)\right\}-\Phi\left\{\left(-\frac{M \sqrt{2 s_{*}}}{\sigma}-\frac{\mu}{\sigma} \sqrt{\frac{2}{s_{*}}}\right)\right\}\right] } \\
& \times \frac{\ln n}{2 \pi}+O(\ln (\ln n)) .
\end{aligned}
$$

Equation (3.58) takes place as above, and from the condition on $\mu / \sigma$, we have

$$
\frac{|\mu|}{\sigma \sqrt{s_{*}}}<\frac{|\mu|}{\sigma \sqrt{\varepsilon}}=\frac{|\mu| / \sigma}{\sqrt{k n^{-1} \ln n}}=O(1) .
$$

Thus, in (3.67), the infinite quantities containing $M$ will outgrow the bounded quantities containing $\mu$, such that $\Phi(+\infty)-\Phi(-\infty)=1$. Therefore

$$
E\{N(0,1-\varepsilon ; M)\} \sim \frac{\ln n}{2 \pi}
$$

3.1.6. Bringing the results together. Combining formulae (3.30), (3.35), and (3.36) shows

$$
E\{N(-\infty,-1+\varepsilon ; M)\}=o(\ln n), \quad E\{N(1-\varepsilon, \infty ; M)\}=o(\ln n) .
$$

That is, the tail sections of the axis contain an asymptotically small number of real $M$ almost zeros. Putting together formulae (3.40) with (3.61), (3.54) with (3.65), and (3.60) with (3.69) for the central section of the axis shows that

$$
E\{N(-1+\varepsilon, 1-\varepsilon ; M)\}=o(\ln n)
$$

in both Cases A and B. However, the conditions of Case C produce

$$
E\{N(-1+\varepsilon, 1-\varepsilon ; M)\} \sim \pi^{-1} \ln n .
$$

Combining these results for the tail and central sections completes the proof of the theorem. 


\section{References}

[1] A. T. Bharucha-Reid and M. Sambandham, Random Polynomials, Probability and Mathematical Statistics, Academic Press, Florida, 1986.

[2] H. Cramér and M. R. Leadbetter, Stationary and Related Stochastic Processes. Sample Function Properties and Their Applications, John Wiley \& Sons, New York, 1967.

[3] J. E. A. Dunnage, The number of real zeros of a class of random algebraic polynomials, Proc. London Math. Soc. (3) 18 (1968), 439-460.

[4] A. Edelman and E. Kostlan, How many zeros of a random polynomial are real? Bull. Amer. Math. Soc. (N.S.) 32 (1995), no. 1, 1-37.

[5] K. Farahmand, Random polynomials with complex coefficients, Statist. Probab. Lett. 27 (1996), no. $4,347-355$.

[6] - Topics in Random Polynomials, Pitman Research Notes in Mathematics Series, vol. 393, Longman, Harlow, 1998.

[7] K. Farahmand and A. Grigorash, Extrema of random algebraic polynomials with non-identically distributed normal coefficients, J. Aust. Math. Soc. 70 (2001), no. 2, 225-233.

[8] J. E. Wilkins Jr., An asymptotic expansion for the expected number of real zeros of a random polynomial, Proc. Amer. Math. Soc. 103 (1988), no. 4, 1249-1258.

[9] Mean number of real zeros of a random trigonometric polynomial, Proc. Amer. Math. Soc. 111 (1991), no. 3, 851-863.

K. Farahmand: School of Computing and Mathematics, Faculty of Engineering, University of Ulster at Jordanstown, Co. Antrim BT37 0QB, UK

E-mail address: k.farahmand@ulster.ac.uk

A. Grigorash: School of Computing and Mathematics, Faculty of Engineering, University of Ulster at Jordanstown, Co. Antrim BT37 0QB, UK

E-mail address: a.grigorash@ulster.ac.uk

P. Flood: School of Computing and Mathematics, Faculty of Engineering, University of Ulster at Jordanstown, Co. Antrim BT37 0QB, UK

E-mail address: floodpaddy@eircom.net 


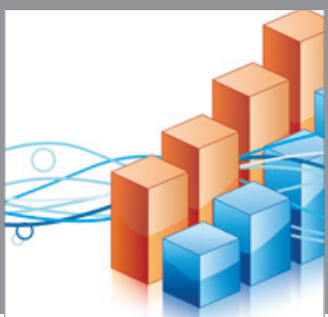

Advances in

Operations Research

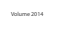

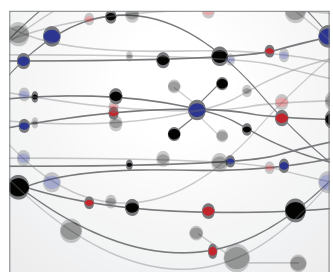

\section{The Scientific} World Journal
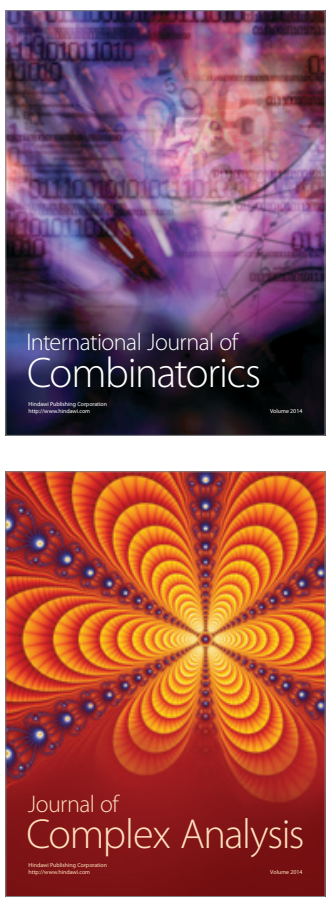

International Journal of

Mathematics and

Mathematical

Sciences
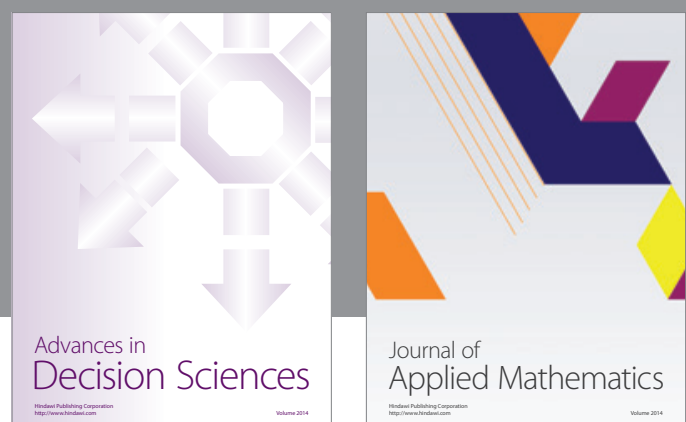

Journal of

Applied Mathematics
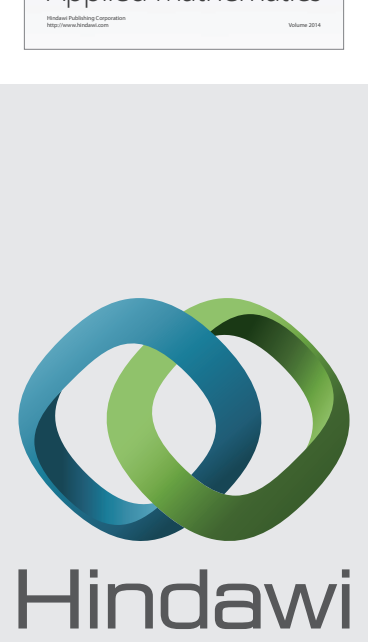

Submit your manuscripts at http://www.hindawi.com
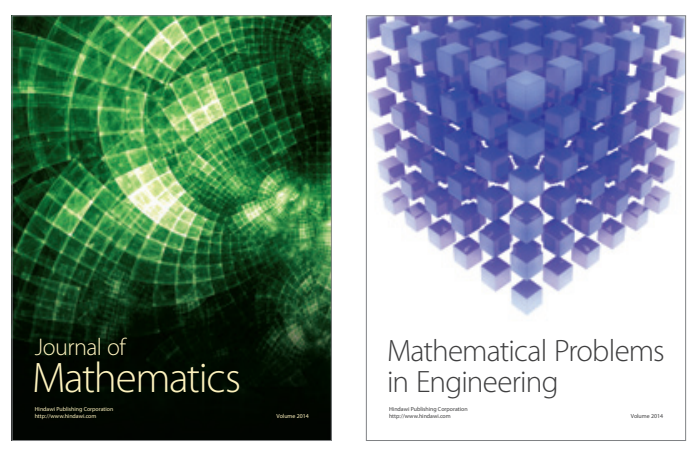

Mathematical Problems in Engineering
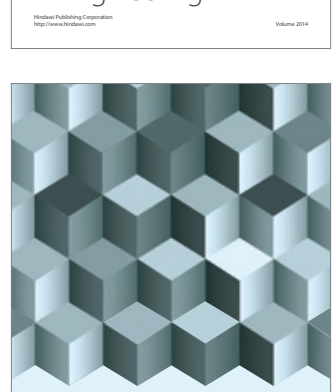

Journal of

Function Spaces
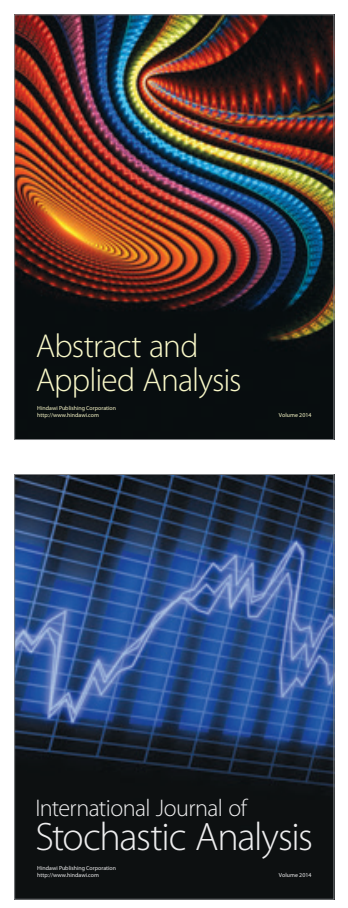

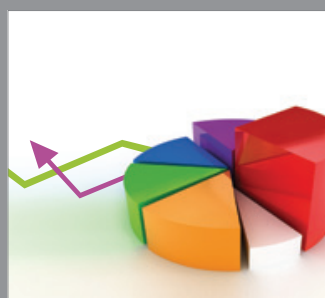

ournal of

Probability and Statistics

Promensencen
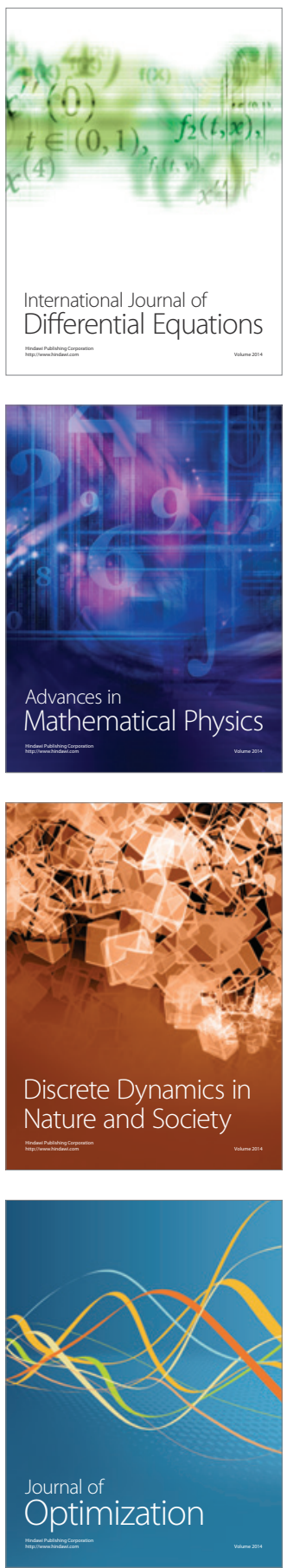